\section{Physician severity scores correlate poorly with health- related quality of life in patients with Hidradenitis Suppurativa}

Dear Editor,

Hidradenitis suppurativa (HS) is known to have a profound impact on the quality of life (QoL) of patients. ${ }^{1}$ Only a few small studies have assessed the Short Form-36 (SF-36) questionnaire

Table 1 Patient characteristics

\begin{tabular}{|c|c|}
\hline & $N=629$ \\
\hline \multicolumn{2}{|l|}{ Sex } \\
\hline Female, $n(\%)$ & 455 (72.3) \\
\hline Age, median [IQR] & $36.0[27.0-46.0]$ \\
\hline Age of onset, median [IQR] & $18.0[15.0-26.0]$ \\
\hline Missing, $n$ & 11 \\
\hline Disease duration, median [IQR] & $12.0[6.0-22.0]$ \\
\hline Missing, $n$ & 11 \\
\hline Body mass index, median [IQR] & $27.2[23.9-31.6]$ \\
\hline Missing, $n$ & 186 \\
\hline \multicolumn{2}{|l|}{ Smoking status } \\
\hline Current or former smoker, $n(\%)$ & $447(71.4)$ \\
\hline Never smoked, $n(\%)$ & $179(28.6)$ \\
\hline Missing, $n$ & 3 \\
\hline \multicolumn{2}{|l|}{ Family history } \\
\hline Positive in 1st or 2st degree, $n(\%)$ & $221(40.8)$ \\
\hline Negative, $n(\%)$ & $252(35.8)$ \\
\hline Unknown, $n(\%)$ & $144(23.3)$ \\
\hline Missing, $n$ & 12 \\
\hline \multicolumn{2}{|l|}{ Comorbidities } \\
\hline Rheumatologic comorbidities, $n(\%)$ & $30(4.8)$ \\
\hline Inflammatory bowel disease, $n(\%)$ & $29(4.6)$ \\
\hline Treated depression, $n(\%)$ & $118(18.8)$ \\
\hline Missing, $n$ & 4 \\
\hline \multicolumn{2}{|l|}{ Hurley stage } \\
\hline $\mathrm{I}, n(\%)$ & $302(53.8)$ \\
\hline II, $n(\%)$ & $220(39.2)$ \\
\hline III, $n(\%)$ & $39(7.0)$ \\
\hline Missing, $n$ & 68 \\
\hline IHS4 & $2.0[0.0-7.0]$ \\
\hline Mild HS ( $\leq 3$ points), $n(\%)$ & $291(55.1)$ \\
\hline Moderate HS (4-10 points), $n(\%)$ & $151(28.6)$ \\
\hline Severe HS ( $\geq 11$ points), $n(\%)$ & $86(16.3)$ \\
\hline Missing, $n$ & 101 \\
\hline
\end{tabular}

Table 1 Continued

\begin{tabular}{ll} 
& $\mathbf{N}=\mathbf{6 2 9}$ \\
\hline NRS pain, median [IQR] & $7.0[4.0-8.0]$ \\
Missing, $n$ & 6 \\
NRS pruritus, median [IQR] & $5.0[2.0-7.0]$ \\
Missing, $n$ & 4 \\
NRS severity, median [IQR] & $7.0[5.0-8.0]$ \\
Missing, $n$ & 3 \\
SF-36 norm-based domain scores & \\
Physical functioning & $46.8(10.6)$ \\
Role physical & $45.2(9.0)$ \\
Bodily pain & $40.6(11.2)$ \\
General health & $40.5(10.8)$ \\
Vitality & $39.9(10.6)$ \\
Social functioning & $41.0(12.6)$ \\
Role emotional & $45.4(9.8)$ \\
Mental health & $42.7(11.7)$ \\
SF-36 component scores & \\
Physical component score & $43.9(9.6)$ \\
Mental component score & $42.1(11.4)$ \\
\hline
\end{tabular}

DLQI, Dermatological life quality index; EQ-5D, Euroqol-5D; IHS4, International Hidradenitis Suppurativa score; NRS, numerical rating scale; SF-36, Short Form 36; VAS, visual analogue scale.

in HS patients. ${ }^{2-5}$ The relation between patient characteristics, patient-reported outcome measures (PROMs) and SF-36 scores has never been evaluated, even though younger age of onset, higher pain and pruritus scores are known to affect other QoL scores among HS patients. ${ }^{5,6}$ The aim of this study was to assess the relation between patient characteristics, PROMs, and objective severity scores and SF-36 scores among HS patients.

All consecutive patients attending the specialized HS clinic of a tertiary centre in the Netherlands between June 2016 and August 2019 were included if they had filled out the SF-36 questionnaire. Patient characteristics, numerical rating scales (NRS) for pain, pruritus, and overall disease severity, Hurley stage, Hidradenitis Suppurativa Severity Score System (IHS4) score and SF-36 scores were collected through the HiScreen Registry (Table 1). Univariate regression analyses were performed for all SF-36 domains (physical functioning, PF; role limitations due to physical health problems, RP; bodily pain, BP; vitality, VT; social functioning, SF; role limitations due to personal or emotional problems, RE; mental health, $\mathrm{MH}$; and general health perceptions, GH) and the physical and mental component scores (PCS, MCS) to inform subsequent multivariate analyses. Multivariate analyses were performed using the forward method with 


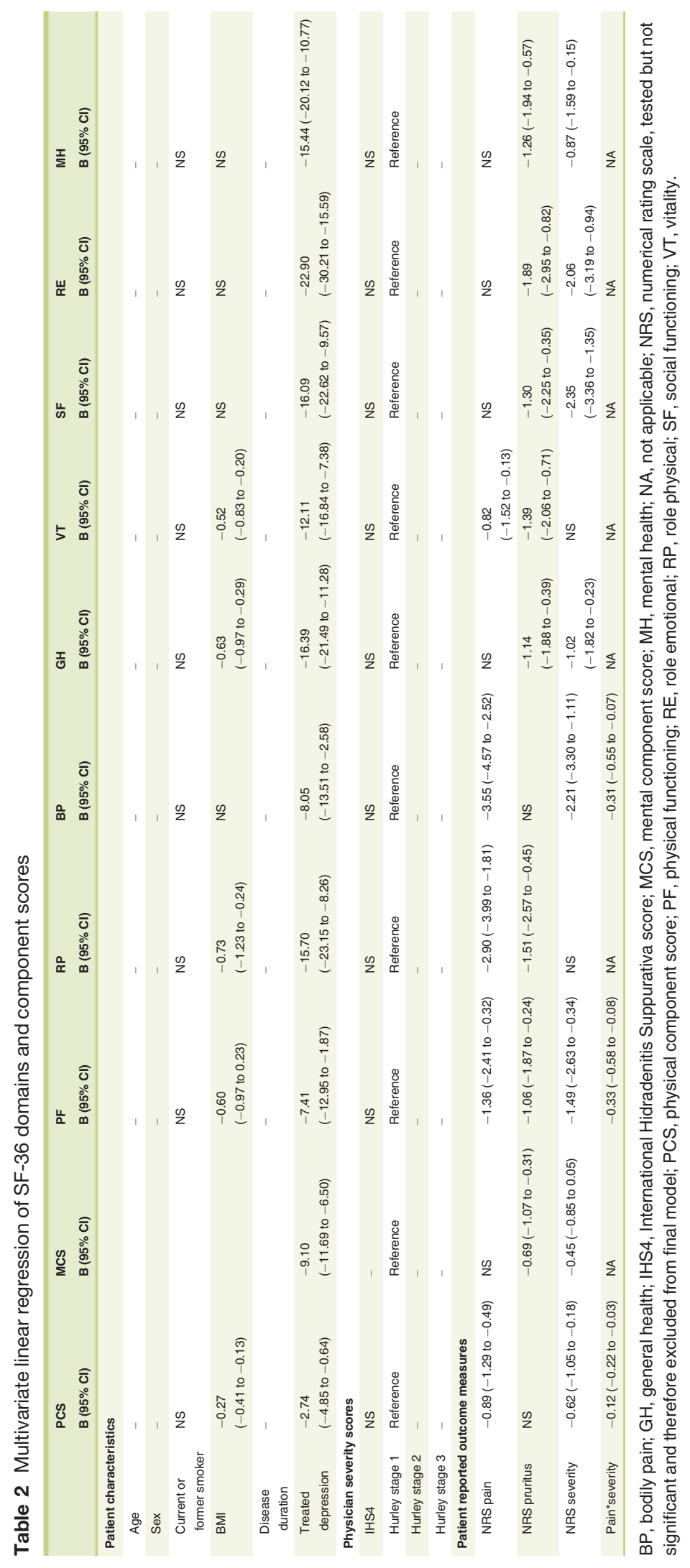


pairwise deletion. Statistical analyses were performed using IBM SPSS Statistics for Windows, version 25.0 (IBM Corp. Armonk, NY, USA).

Multivariate analysis showed treated depression as the largest factor influencing the PCS and MCS, respectively $\mathrm{B}=-2.74,95 \% \mathrm{CI}-4.85$ to -0.64 and $\mathrm{B}=-9.10,95 \% \mathrm{CI}$ -11.69 to -6.50 , and each of the individual SF-36 domains (Table 2). None of the physician scores were significantly correlated with any of the domains or the component scores. NRS pain was significant in the physical domains (PF, RP, BP and $\mathrm{VT}$ ), but in not in the GH, SF, RE and $\mathrm{MH}$ domains. Body mass index was a significant predictor of the PF, RP, GH and VT domains, but not of the SF, RE and MH domains. NRS pruritus was significantly associated with all individual domains except for BP.

This is the first study to assess the contribution of patient characteristics, PROMs and severity scores to SF-36 scores in a large cohort of HS patients. The mean component scores found in our study are in line with those reported by Kolli et al., 40.9 (6.3) and 40.0 (6.4), and those found by Hamzavi et al., 39.6 (9.4) and $41.5(12.40)^{3,4}$ Treated depression was the most important predictor for a lower score for both the MCS and PCS and each individual domain. This emphasizes the high burden of depression among HS patients on both psychological and physical domains. ${ }^{7}$ Our results do not support an independent relation between Hurley stage or IHS4 score for the component scores or the domains scores. This might be due to the generic nature of the SF-36 as it does not include specific HS related quality of life problems, for example discomfort from drainage or foul smell. A disease-specific QoL questionnaire could include these important aspects and could be more sensitive to disease severity. ${ }^{8}$

In conclusion, HS severity scores were not associated with QoL, and treated depression was the largest independent factor for both component scores and all SF-36 domains. These results suggest that the generic SF-36 does not accurately capture quality of life impairment due to HS. Moreover, comorbid depression should be taken into account and corrected for when analysing quality of life in HS patients.

\section{Funding sources}

None.

$$
\begin{aligned}
& \text { K.R. van Straalen, * iD I. van Trigt, iD E.P. Prens, iD } \\
& \text { H.H. van der Zee id }
\end{aligned}
$$

\section{References}

1 Jemec GBE, Heidenheim M, Nielsen NH. The prevalence of hidradenitis suppurativa and its potential precursor lesions. J Am Acad Dermatol 1996; 35: 191-194.

2 Alavi A, Anooshirvani N, Kim WB, Coutts P, Sibbald RG. Quality-of-life impairment in patients with hidradenitis suppurativa: a Canadian study. Am J Clin Dermatol 2015; 16: 61-65.

3 Hamzavi IH, Sundaram M, Nicholson Cet al. Uncovering burden disparity: a comparative analysis of the impact of moderate-to-severe psoriasis and hidradenitis suppurativa. J Am Acad Dermatol 2017; 77: 1038-1046.

4 Kolli SS, Senthilnathan A, Cardwell LAet al. Hidradenitis suppurativa has an enormous impact on patients' lives. J Am Acad Dermatol 2019; 82: 236238.

5 Wolkenstein P, Loundou A, Barrau Ket al. Quality of life impairment in hidradenitis suppurativa: a study of 61 cases. J Am Acad Dermatol 2007; 56: 621-623.

6 Riis PT, Vinding GR, Ring HC, Jemec GBE. Disutility in patients with hidradenitis suppurativa: a cross-sectional study using EuroQoL-5D. Acta Dermato Venereol 2016; 96: 222-226.

7 Machado MO, Stergiopoulos V, Maes Met al. Depression and anxiety in adults with hidradenitis suppurativa: a systematic review and meta-analysis. JAMA Dermatol 2019; 155: 939.

8 Kirby J.S., Thorlacius L., Villumsen B., Ingram J.R., Garg A., Christensen K.B., Butt M., Esmann S., Tan J., Jemec G.B.E.. The Hidradenitis Suppurativa Quality of Life (HiSQOL) score: development and validation of a measure for clinical trials. British Journal of Dermatology. 2019; http://dx.doi. org/10.1111/bjd.18692.

DOI: $10.1111 / j d v .16541$ 